\title{
Lumen
}

Selected Proceedings from the Canadian Society for Eighteenth-Century Studies

\section{Les secrets plaisirs de la voyeuse au temps des Lumières}

\section{Florence Fesneau}

Volume 37, 2018

URI : https://id.erudit.org/iderudit/1042230ar

DOI : https://doi.org/10.7202/1042230ar

Aller au sommaire du numéro

Éditeur(s)

Canadian Society for Eighteenth-Century Studies / Société canadienne d'étude du dix-huitième siècle

ISSN

1209-3696 (imprimé)

1927-8284 (numérique)

Découvrir la revue

Citer cet article

Fesneau, F. (2018). Les secrets plaisirs de la voyeuse au temps des Lumières.

Lumen, 37, 175-198. https://doi.org/10.7202/1042230ar d'utilisation que vous pouvez consulter en ligne.

https://apropos.erudit.org/fr/usagers/politique-dutilisation/ 


\title{
Les secrets plaisirs de la voyeuse au temps des Lumières
}

\author{
Florence Fesneau \\ Université Paris 1 Panthéon-Sorbonne
}

\begin{abstract}
À la veille de la Révolution, dans ses Nuits de Paris ${ }^{1}$, Restif raconte que son spectateur nocturne se voit proposer par un «Falot ${ }^{2}$ » un «lit de passade» dans un hôtel borgne. Mais la nuit qu'il devait passer avec deux jeunesses est interrompue par la querelle de ses voisins de chambre qu'il observe incognito en soulevant un tableau, tandis que lui-même est soumis aux regards inquisiteurs de deux voyeurs (Fig. 1). Lillustration qui accompagne ce texte met en exergue - ici de manière comiquement caricaturale - une activité souvent dépeinte dans la peinture de genre au XVIII ${ }^{\mathrm{e}}$ siècle; celle du voyeur. C'est sur l'illustration de ce voyeurisme que la présente étude souhaite s'interroger et plus particulièrement sur les rapports entre l'activité bien connue du voyeur ${ }^{3}$ et celle - moins souvent étudiée - de la voyeuse. Cette voyeuse - telle que décrite et illustrée au travers de la gravure des nouvelles ou dans la peinture - se définit-elle de manière identique ou différente de son équivalent masculin? Et que peut-elle nous révéler du statut de la femme au XVIII siècle?
\end{abstract}

1. Nicolas-Edme Restif de La Bretonne, Les nuits de Paris, ou le spectateur nocturne. Quatrième partie, Londres, s. n., 1788-1794, p. 814-821.

2. Un porteur de lanterne.

3. Trop nombreux sont les auteurs à avoir abordé ce sujet pour être tous cités, mais l'on retiendra les textes séminaux d'Henri Lafon, «Voir sans être vu : un cliché, un fantasme », dans Poétique, 29, 1977, p. 50-6o et de Catherine Cusset, «Le voyeurisme des Lumières », dans Studies on Voltaire and the Eighteenth Century, 347, 1996, p. $831-833$. 
176 Florence Fesneau

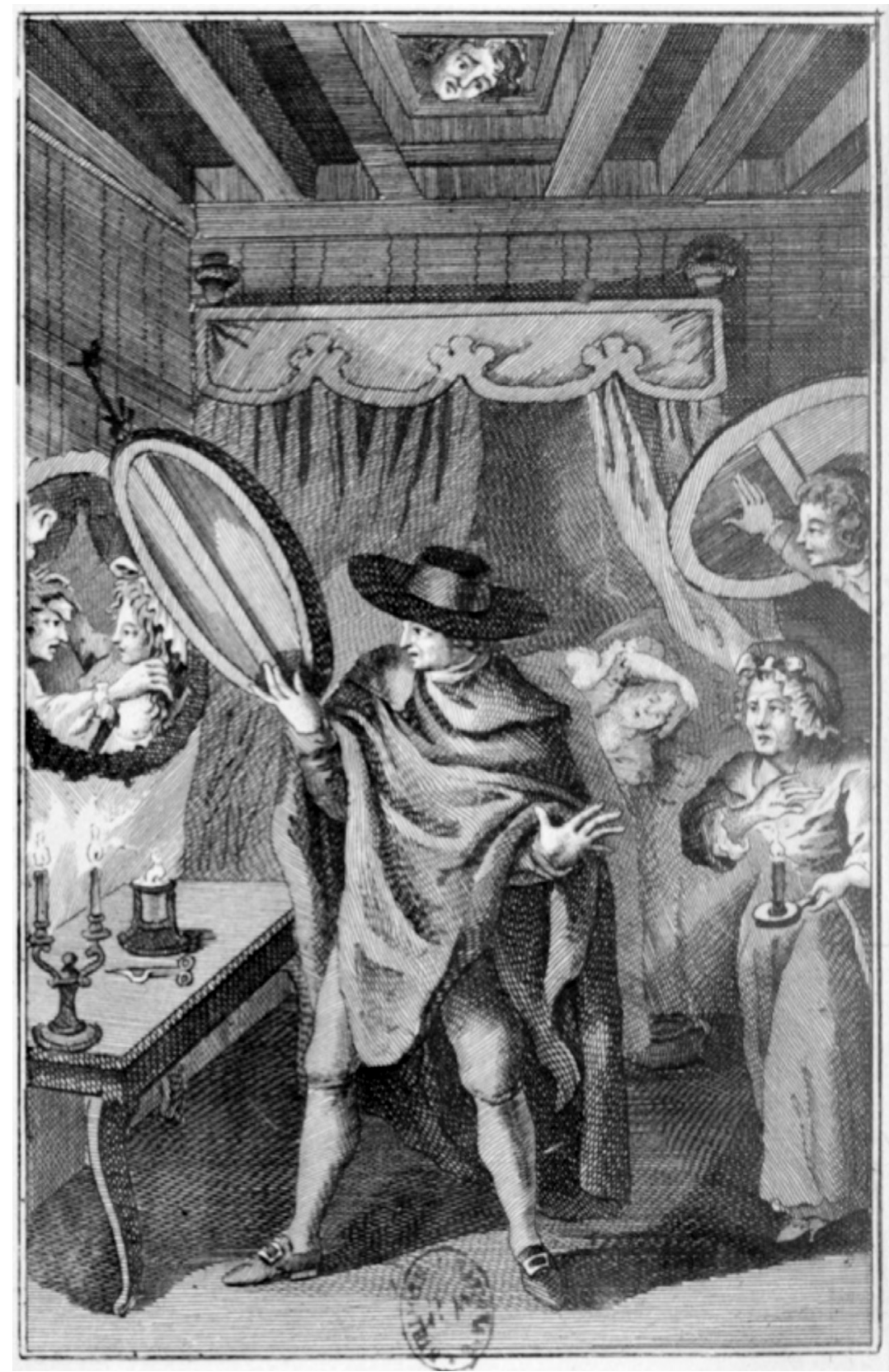

Fig. 1: Anonyme, Frontispice du t. IV, dans Restif de La Bretonne, NicolasEdme, Les nuits de Paris, ou Le spectateur nocturne, Londres, s. n., 1788, t. IV. 


\section{Voyer, voyeur et voyeuse}

Mais peut-être faut-il commencer par un point de vocabulaire pour souligner que si les tableaux et gravures du XviII ${ }^{\mathrm{e}}$ siècle sont hantés par la figure du voyeur, celui-ci n'existe pas. Tout du moins en tant que nom désignant une personne qui aime regarder les choses et les gens et, par extension, quelqu'un qui aime observer l'intimité des autres à leur insu. Le Dictionnaire universel de Furetière ${ }^{4}$ et celui de Richelet ${ }^{5}$ ne connaissent que le «Voyer». Ce mot proche à l'oreille de celui de «Voyeur»- le Dictionnaire de Trévoux ${ }^{6}$ met d'ailleurs en garde contre cette prononciation abusive - se rattache étymologiquement à la voirie, la charge de Grand Voyer de France ayant été créée pour Sully. Le terme de « $\operatorname{Voyeur}^{7} »$, au sens où on l'entend aujourd'hui, sera utilisé pour la première fois par Alphonse Daudet en $1898^{8}$ et le «Voyeurisme » ne fera son entrée lexicale que tardivement, en 1955, sous la plume du psychologue Henri Piéron?.

En revanche la «Voyeuse» existe bel et bien au XVIII ${ }^{\mathrm{e}}$ siècle. Mais il s'agit d'un siège représenté sur la planche XIV consacrée au «Tapissier » dans l'Encyclopédie de Diderot et D’Alembert. La planche est ainsi légendée: «Voyeuse, espèce de chaise pour s'asseoir à cheval \& s'accouder sur le dossier pour voir jouer. » La voyeuse ${ }^{10}$, aussi appelée « voyeuse à genou », est souvent placée dans les salons de jeux pour permettre aux spectateurs de suivre, par-dessus l'épaule des joueurs, les parties de cavagnole ${ }^{11}$, de

4. Antoine Furetière, Dictionnaire universel, La Haye, Leers, 1701 [169o], t. III, p. 1091, Art. «Voyer».

5. Pierre Richelet, Dictionnaire françois, Amsterdam, Elzevir, 1706 [1680], p. 886, Art. «Voïer».

6. Dictionnaire universel françois et latin: vulgairement appelé Dictionnaire de Trévoux, Paris, Compagnie des Libraires Associés, 1771 [1704], t. VIII, p. 476, Art. «Voyer».

7. L'origine du mot - elle proviendrait de l'ancien français veor - reste tout aussi obscure que l'activité cachée que ce mot décrit.

8. Alphonse Daudet, Soutien de famille, mours contemporaines, Paris, Fasquelle, 1898 , p. 136 , chap. 2 «Fin de bal».

9. Henri-Louis-Charles Piéron (1881-1964), psychologue français, fondateur de la psychologie scientifique en France.

10. Il semblerait que ce soit le menuisier en sièges Jean-Baptiste-Claude Séné (1748-1803) ou l'ébéniste Georges Jacob (1739-1814) qui l'ait inventée.

11. Jeu d'origine génoise, introduit en France dans les années 1730, surtout joué par les femmes et assimilable à une loterie où l'on parie sur les numéros qui vont sortir. 
papillon ${ }^{12}$ ou de pharaon ${ }^{13}$. Les spectateurs et spectatrices s'installent sur une voyeuse, soit à genoux - comme sur un prie-Dieu - en prenant appui sur l'accoudoir rembourré, soit à califourchon, position néanmoins réservée exclusivement aux hommes.

\section{Voyeurs, lorgneurs, indiscrets et curieux}

Repérer la figure du voyeur ou de la voyeuse dans les tableaux et gravures qui les mettent en scène, n'est donc pas toujours chose aisée puisqu'ils ne sont pas nommés et que, de plus, ils se cachent souvent à l'arrière-plan, jusqu'à tenter d'être indétectables. Cependant le voyeur - qui s'ignore encore comme tel - peut alternativement être qualifié d'indiscret, de curieux ou de lorgneur. Avant de procéder plus avant, il est nécessaire de voir si ces notions ont une valeur similaire à celle de voyeur et de s'assurer de l'intérêt qu'elles suscitent.

On rappellera que le XVII siècle a plutôt une approche dépréciative de la curiosité. Dans ses Réflexions, l’abbé de Bellegarde fustige l'indiscrétion et observe: "Il est permis de tout voir, quand on a de bons yeux; mais il n'en faut rien témoigner, si l'on a soin de son repos. Quand on a tout entendu, il faut avoir la discrétion de se taire ${ }^{14}$. " Mais dès le début du XVIII ${ }^{\mathrm{e}}$ siècle se répandent les lorgneurs et les lorgneuses qui vont poser des regards de plus en plus indiscrets sur ce qui les entoure: dans deux tableaux en pendant datant de $1716^{15}$, Watteau montre d'une part un mezzetin donnant une aubade à une jeune femme assise à ses pieds, tout en lorgnant sur son décolleté, et, d'autre part, la scène inverse, à savoir une jeune-femme lorgnant sur un joueur de flute assis à ses côtés. Cette pratique est si bien ancrée dans les

12. Se joue à trois avec un jeu de 52 cartes.

13. Jeu de cartes qui se déroule entre un banquier et un nombre indéfini de joueurs appelés «pontes» sur une table ovale recouverte de feutrine verte.

14. Jean-Baptiste Morvan de Bellegarde, Réflexions sur le ridicule et sur les moyens de l'éviter, où sont représentez les mœurs et les différens caractères des personnes de ce siècle, Paris, Guignard, 1700 [1697], p. 46.

15. Le lorgneur, 1716, huile sur bois, $32,4 \times 23,9 \mathrm{~cm}$, Richmond, Virginia Museum of Fine Arts, et La lorgneuse, 1716, huile sur bois, 32,6 ×23,8 cm, marché de l'art. Les titres des tableaux sont attestés par les gravures de Jean-Baptiste Scotin d'après Watteau, Le lorgneur et La lorgneuse, 1726, gravures, $38,7 \times 28,5 \mathrm{~cm}$ (chacune), Paris, Bibliothèque nationale de France. 
habitudes parisiennes que Mercier y consacre un chapitre dans ses Tableaux de Paris en $1781^{16}$.

Quant à l'indiscrétion voyeuriste, elle n'est plus l'apanage du seul rustre des campagnes - comme semble le suggérer Watteau, qui montre un paysan glissant un regard subreptice sous les jupes d'une fileuse de laine ${ }^{17}$ - mais se répand progressivement - et s'image - dans la population urbaine. Le XVIII ${ }^{\mathrm{e}}$ siècle va ainsi mettre les actions des curieux et des indiscrets en exergue. Ceci est perceptible aussi bien dans les gravures que dans les romans et les pièces de théâtre, leurs auteurs faisant du curieux ou de l'indiscret non seulement un de leurs personnages favoris, mais allant même jusqu'à donner à ce nouveau héros le titre de leur œuvre. Dans Le curieux impertinent ${ }^{18}$ et L'ambitieux et L'indiscrète ${ }^{19}$ de Destouches, comme dans L'indiscret de Voltaire ${ }^{20}$, il ne s'agit encore que de conversations entendues par des oreilles indiscrètes. Mais, en 1748, dans Les bijoux indiscrets de Diderot, le sultan Mangogul se voit doter d'un pouvoir d'indiscrétion qui lui permet de percer les pensées les plus intimes des gens de sa cour. Grâce à la bague que lui donne le génie Cucufa, il peut faire parler les «bijoux» des femmes comme bon lui semble, dont ceux d'Haria, que Mangogul surprend pendant son sommeil ${ }^{21}$.

Juste avant la Révolution, ce sont deux femmes de chambre L'indiscrette $^{22}$ et La curieuse impertinente ${ }^{23}$ - qui relatent leurs expériences de voyeuses dans des romans érotiques restés anonymes. Bien qu'antérieure à ces deux romans, la gouache de Pierre-Antoine Baudouin ${ }^{24}$ pourrait parfaitement venir les illustrer. Les deux gravures

16. Louis-Sébastien Mercier, Tableaux de Paris, Hambourg/Neuchatel, Virchaux \& Cie/Fauche, 1781 1, t. I, p. 203, chap. CLXI.

17. Jean-Antoine Watteau, Lindiscret, 1716, huile sur bois, 21,6×25,9 cm, Rotterdam, Museum Boijmans Van Beuningen. Le tableau est repris en gravure par Michel Guillaume Aubert, Lindiscret, 1725, gravure, 41,6 × 46,7 cm, Paris, Bibliothèque nationale de France.

18. Philippe Néricault Destouches, Le curieux impertinent, comédie en cinq actes, représentée le 17 décembre 1710.

19. Philippe Néricault Destouches, L'ambitieux et l'indiscrète, tragi-comédie, représentée le 14 juin 1737 .

20. François-Marie Arouet dit Voltaire, L'indiscret, comédie, représentée le $1^{\mathrm{er}}$ août 1725 .

21. Denis Diderot, Les bijoux indiscrets, Au Monomotapa, s. n., 1748, t. I., p. 232.

22. L'indiscrette ou Mémoires d'une femme-de-chambre, Londres, s. n., 1787, 2 t.

23. La curieuse impertinente. Traduite de l'anglois, s. 1., s. n., 1789, 2 t.

24. L'épouse indiscrète, 1768 , gouache, $30 \times 27 \mathrm{~cm}$, Paris, Musée des arts décoratifs. 
qui en ont été tirées, par Nicolas Delaunay en 1771, s'intitulent alternativement L'épouse indiscrète ou Les indiscrets. Elles mettent l'accent soit sur la voyeuse qui semble plus curieuse d'assister aux ébats du couple adultère que de s'indigner de l'infidélité de son mari (si tel est bien le statut de cet homme), soit sur les amants illicites, absorbés dans leurs jeux amoureux, et fort oublieux d'observer les règles élémentaires de discrétion en pareilles circonstances.

\section{Choses vues}

L'intérêt que suscitent les agissements des curieux et des curieuses au XVIII ${ }^{\mathrm{e}}$ siècle n'étant plus à démontrer, il s'agit maintenant de qualifier le spectacle qui retient toute leur attention ou, plus exactement, de savoir si le voyeur et la voyeuse regardent la même chose. À cette question, on serait tenté de répondre par la négative, mais la réponse mérite d'être nuancée.

Bon nombre de situations semblent être effectivement réservées au seul voyeur, à quelques exceptions près. En premier lieu, le thème du bain en plein air - qu'il soit traité par Jean-Baptiste Pater, Nicolas Lancret, François Boucher, ou plus tardivement par Jean-Frédéric Schall - met en présence des baigneuses plus ou moins dévêtues avec des voyeurs cachés dans les fourrés environnants. Linverse est inexistant dans la peinture de genre. Tout au plus peut-on citer les deux gravures de 1776 (Fig. 2) et $1785^{25}$ illustrant le baptême de l'Ingénu sous le regard intéressé des demoiselles de Kerkabon et de Saint-Yves, qui aperçoivent le Huron nu dans la rivière. Voltaire précise que ces dernières «jetèrent un grand cri, et se détournèrent. Mais la curiosité l'emportant bientôt sur toute autre considération, elles se coulèrent doucement entre les roseaux; et quand elles furent sûres de n'être point vues, elles voulurent voir de quoi il s'agissait ${ }^{26}$.»

De même, le thème de la toilette féminine est-il très favorable au voyeur masculin qu'il n'est pas difficile de débusquer à l'arrièreplan des tableaux et gravures de Pater, Baudouin ou Antoine Borel.

25. Jean-Baptiste Simonet d'après Jean-Michel Moreau, L'ingénu baptisé, 1782, gravure, dans Voltaire, Euvres complètes, Paris, Société littéraire typographique, 1785 , t. XLIV, p. 364.

26. Voltaire, op. cit., t. XLIV, p. 363: chap. III, Le Huron, nommé l'Ingénu, converti. 


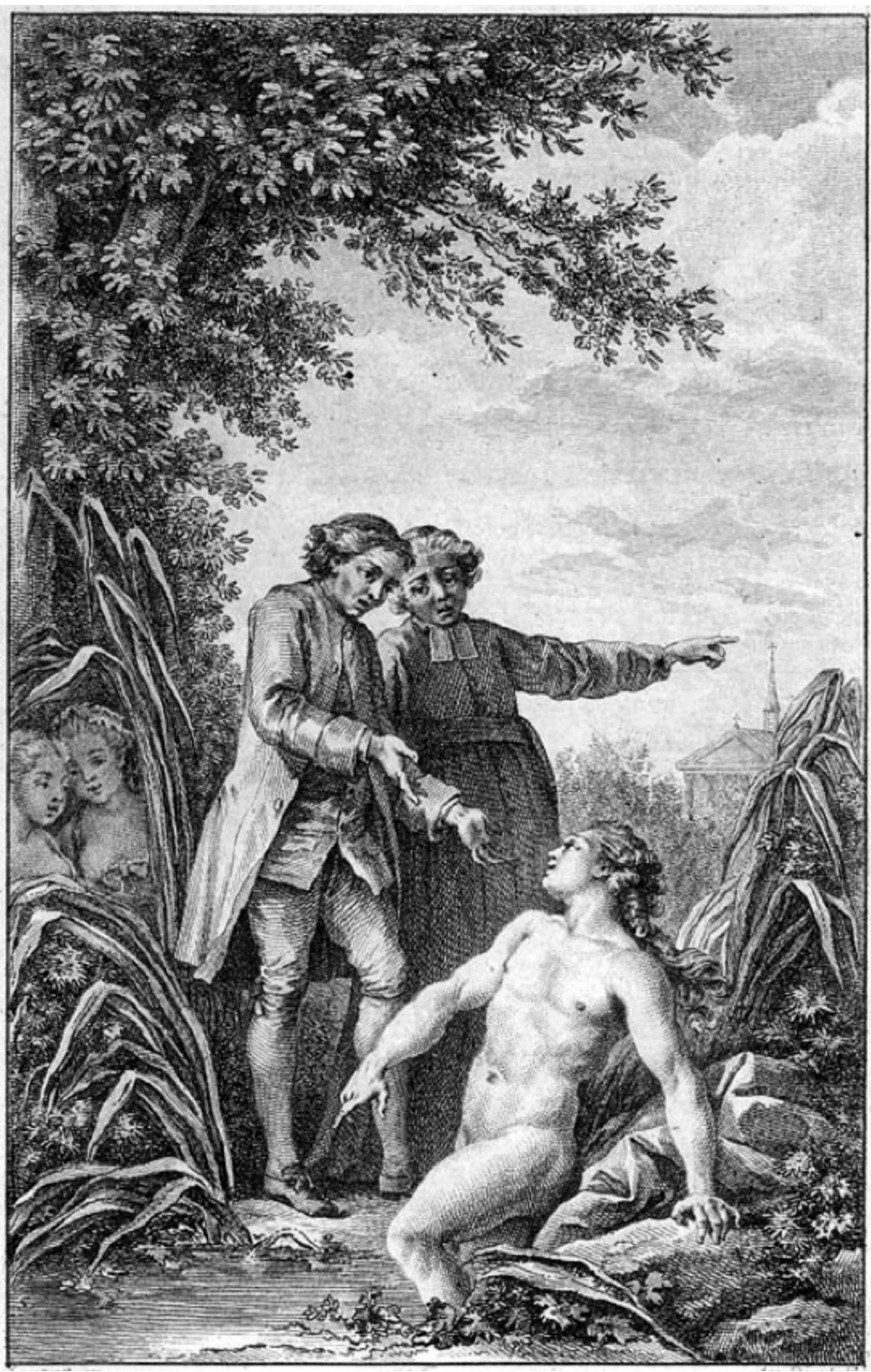

Fig. 2: Jeanne Deny d'après Charles Monnet, Lingénu baptisé, gravure, dans François-Marie Arouet dit Voltaire, Romans et contes, Bouillon, Société typographique, 1776 , t. II, en regard de la p. 234. 


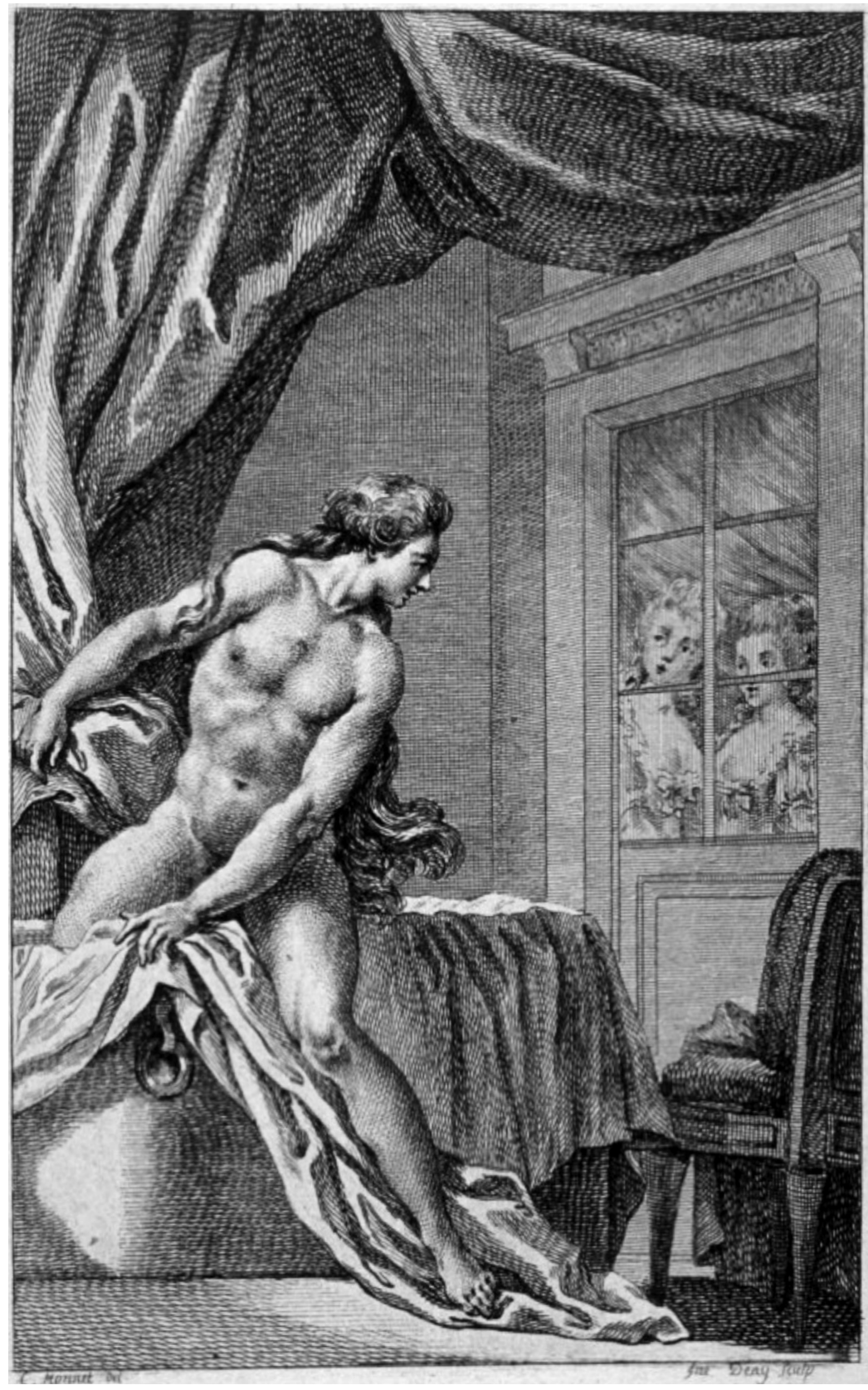

Fig. 3: Jeanne Deny d'après Charles Monnet, Jeune homme à sa toilette, gravure, dans François-Marie Arouet dit Voltaire, Romans et contes, Bouillon, Société typographique, 1778 , t. III, Jenni ou Le sage et l'athée, en regard de la p. 7. 
L'illustration (Fig. 3) d'une nouvelle de Voltaire, Jenni ou Le sage et l'athée, fournit à nouveau un rare contre-exemple dans lequel deux espagnoles de Gibraltar brulent d'impatience à l'idée de voir à quoi ressemble le prisonnier anglais qui, comme tous ces «English hérétiques» devait avoir «des queues de singes, des pattes d'ours, \& des têtes de perroquets". Elles se cachent donc «dans une petite garderobe, derrière une jalousie par laquelle on voyait la baignoire [...] dans le temps où l'English sortait de l'eau ${ }^{27}{ }$. Et elles sont fort agréablement surprises, au point de révéler involontairement leur présence par leurs commentaires émus.

Troisième sujet de voyeurisme typiquement masculin; l'administration du clystère, un remède, qui, pour L'élite de bons mots, "peut [pour les femmes] conserver de leur teint la fraîcheur // Dans une vive $\&$ charmante couleur ${ }^{28}{ }$. Le remède de Watteau ${ }^{29}$, comme le Curieux de Baudouin ${ }^{30}$, la Soubrette officieuse de Schallil et le Contretemps de Lavreince $^{32}$, déguisent malicieusement le propos graveleux ${ }^{33}$ de ces scènes dans lesquelles le voyeur assiste à l'événement, avec la complicité de la soubrette officiant auprès d'une maîtresse qui ignore être observée. De toute évidence, il est impossible de trouver l'équivalent, au féminin, de ces images, la femme restant - au mieux - cantonnée dans un rôle de garde malade, comme le montre l'illustration du Malade imaginaire (Fig. 4) aux pieds duquel se trouvent diverses fioles et cataplasmes accompagnés d'un clystère.

27. Voltaire, Romans et contes, Bouillon, Société typographique, 1778, t. III, p. 6 : Jenni ou Le sage et l'athée.

28. Élite de bons mots, Amsterdam, Mortier, t. II, 1745, p. 365-367: Le clystère.

29. 1716-1717, dessin, 23,3 × 37,1 cm, Los Angeles, J. Paul Getty Museum.

30. Pierre Maleuvre d'après Pierre-Antoine Baudouin, Le curieux, 1763-1769, gravure, $33 \times 27 \mathrm{~cm}$, Paris, Bibliothèque nationale de Paris.

31. Alexandre Chaponnier d'après Jean-Frédéric Schall, La soubrette officieuse, après 1785 , gravure, $32 \times 36 \mathrm{~cm}$, marché de l'art.

32. François-Nicolas Barthélémy Dequevauvillier d'après Nicolas Lavreince, Le contretemps, 1786, gravure, Washington, National Gallery of Art.

33. Mary L. Bellhouse, "Erotic "remedy" prints and the fall of the aristocracy in eighteenth-century France", dans Political Theory, 25, 5, octobre 1997, p. 680-715; Donald Posner, "Watteau's reclining nude and the remedy Theme", dans The Art Bulletin, 54, 4, décembre 1972, p. 383-389. 
$184 \$$ Florence Fesneau

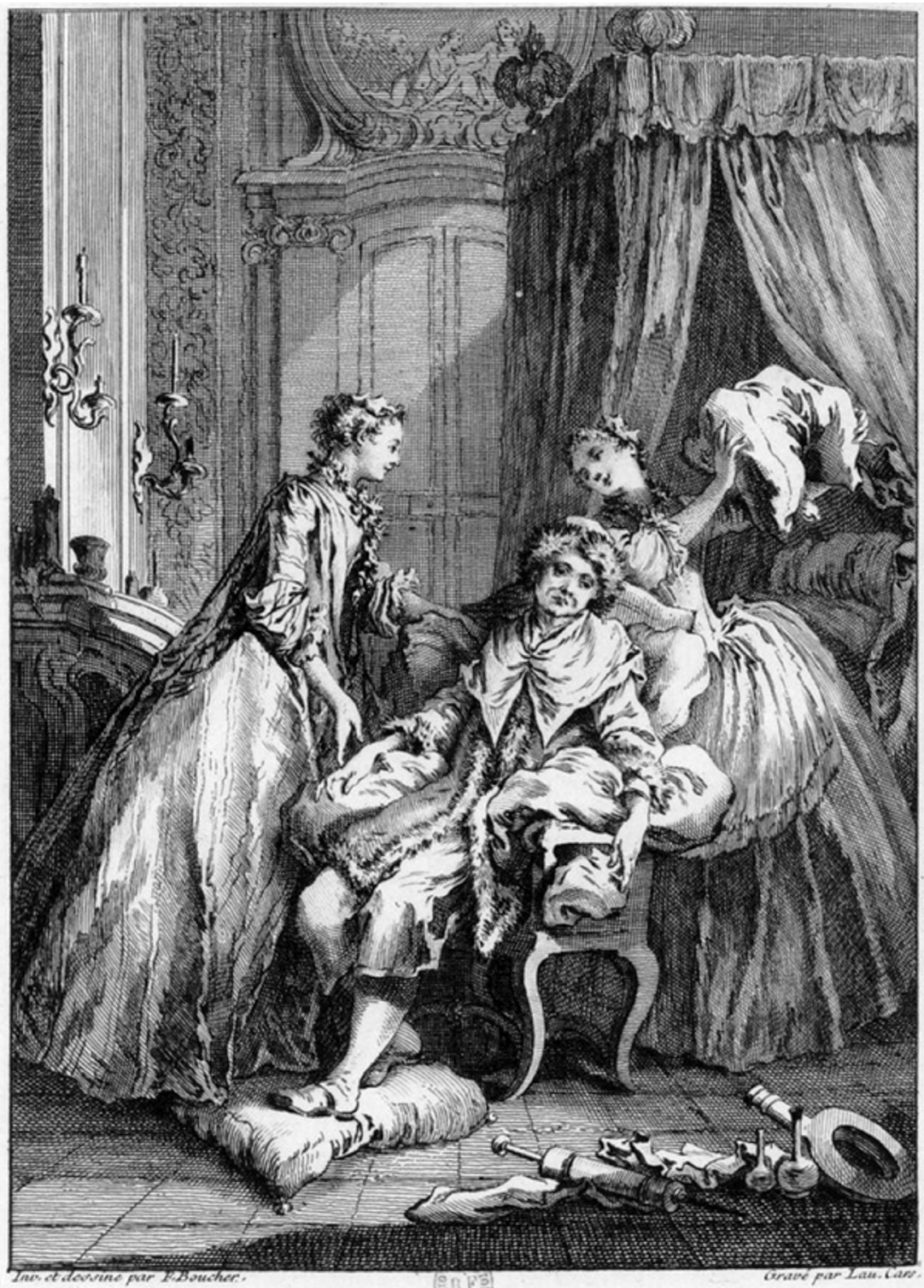

LE MALADE IMAGINAIRE

Fig. 4: Laurent Cars d'après François Boucher, Le malade imaginaire, gravure, dans Jean-Baptiste Poquelin dit Molière, Euvres, nouvelle édition, Paris, [David?], 1734, t. VI, en regard de la p. 378. 


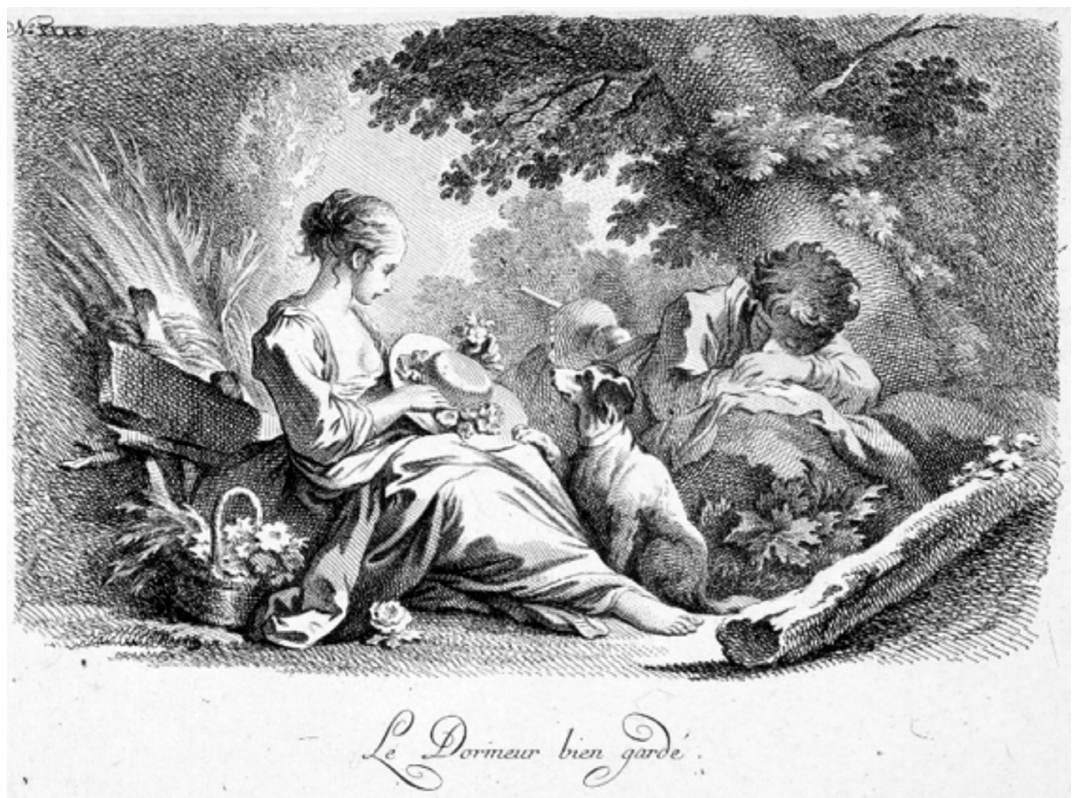

Fig. 5: Gilles Demarteau d'après François Boucher, Le dormeur bien gardé, 1741-1788, gravure, 16,7 x 22,2 cm, Brunswick, Herzog Anton Ulrich-Museum.

\section{Voyeurs et dormeurs}

Les situations évoquées semblent indiquer clairement qu'être voyeur est une activité qui se vit et s'image essentiellement au masculin. Dorat rappelle d'ailleurs, dans sa Fable sur Le secret de l'éducation, que l'indiscrétion voyeuriste sied mal à la gente féminine. Il explique comment guérir la «curiosité la plus incorrigible» de celle qui «voulait tout voir, tout épier» en «s'attachant au trou de la serrure ${ }^{34}$ ». Toutefois le sommeil du dormeur ou de la dormeuse, épiés - par définition - à leur insu, offrent une série de situations permettant de figurer une voyeuse qui peut avoir des comportements similaires à ceux du voyeur, sans en avoir néanmoins toutes les prérogatives.

Dans le Dormeur bien gardé de François Boucher (Fig. 5), la voyeuse regarde sagement le berger endormi et semble prête à déposer à ses pieds un bouquet de fleurs. En cela elle ne diffère guère des

34. Claude-Joseph Dorat, Fables ou allégories philosophiques, Paris, Delalain, 1772, p. 10. 
galants du Panier mystérieux $x^{35}$ et de La jardinière endormie ${ }^{36}$ du même François Boucher, qui viennent déposer leur présent aux pieds de l'amante assoupie. En revanche, si le voyeur peut profiter de la situation pour dérober à la dormeuse sa cage - voire, symboliquement, bien plus encore - comme le montre le tableau de Noël Hallé37, la voyeuse - au contraire - renforce les signes de son passage en laissant des témoignages de son émerveillement dédicatoire: un ruban pour John Gay ${ }^{38}$ ou un mouchoir de col pour Favart dans Les moissonneurs (Fig. 6) afin de protéger le dormeur du soleil.

La voyeuse est rarement aussi téméraire que le voyeur, qui n’hésite pas à pénétrer au sein d'un jardin privé, tel que le montre Jean-Michel Moreau dans sa Dormeuse ${ }^{39}$, ou à s'aventurer jusqu'aux portes du boudoir ${ }^{40}$ ou de la chambre de la belle endormie. La gravure d'AntoineJean Duclos ${ }^{41}$ illustre le moment où le Chevalier de Versenai est saisi du « désir coupable de voir et d'admirer pendant son repos» Madame de Senanges. Dans sa lettre à son ami le Baron, il se défend de toute mauvaise intention: "J'étais immobile d'admiration \& de plaisir; je n'entrevoyais pas même la possibilité d'attenter à ses charmes. C'était mon âme qui jouissait; mes sens étaient enchainés par le respect, \& je m’étais prosterné devant cet ange dont je n'osais m'approcher ${ }^{42}$.» Mais le voyeur - contrairement à la voyeuse - n'est pas toujours aussi respectueux du sommeil de l'endormie. Il peut facilement devenir un prédateur à l'instar de Vicomte de Valmont qui s'introduit dans la chambre de Cécile Volanges, comme le montre la gravure de Masquelier ${ }^{43}$ accompagnant le texte : «Elle était dans son premier

35. 1748, huile sur toile, $92,7 \times 78,9 \mathrm{~cm}$, Melbourne, National Gallery of Victoria. 36. 1762 , huile sur toile, $232 \times 91 \mathrm{~cm}$, Édimbourg, The National Galleries of Scotland.

37. La cage dérobée, 1763 , huile sur bois, $18,4 \times 25,3 \mathrm{~cm}$, collection particulière.

38. John Gay, The Shepherd's Week in six Pastorals, Londres, Burleigh, 1714, p. 30-38: «Thursday or the Spell».

39. 1774, gravure, dans Jean-Benjamin de Laborde, Choix de chansons mises en musique, Rouen, Lemonnyer, 1881 [1774], t. I, p. 24.

40. Pierre Maleuvre d'après Sigismond Freudeberg, Le boudoir, 1774, gravure, dans Histoire des mœurs et du costume des Français dans le XVIII siècle, Paris, Prault, 1775.

41. D'après Clément-Pierre Marillier, 1772, gravure, dans Claude-Joseph Dorat, Les sacrifices de l'amour, Amsterdam/Paris, Delalain.

42. Claude-Joseph Dorat, op. cit., t. I, p. 298.

43. D’après Marguerite Gérard, Lettre XCVI, 1796, Planche VII dans PierreAmbroise-François Choderlos de Laclos, Les Liaisons dangereuses. Texte intégral d'après l'édition de 1782, Paris, Bibliothèque des curieux, 1913. 
Les secrets plaisirs de la voyeuse au temps des Lumières \$ 187

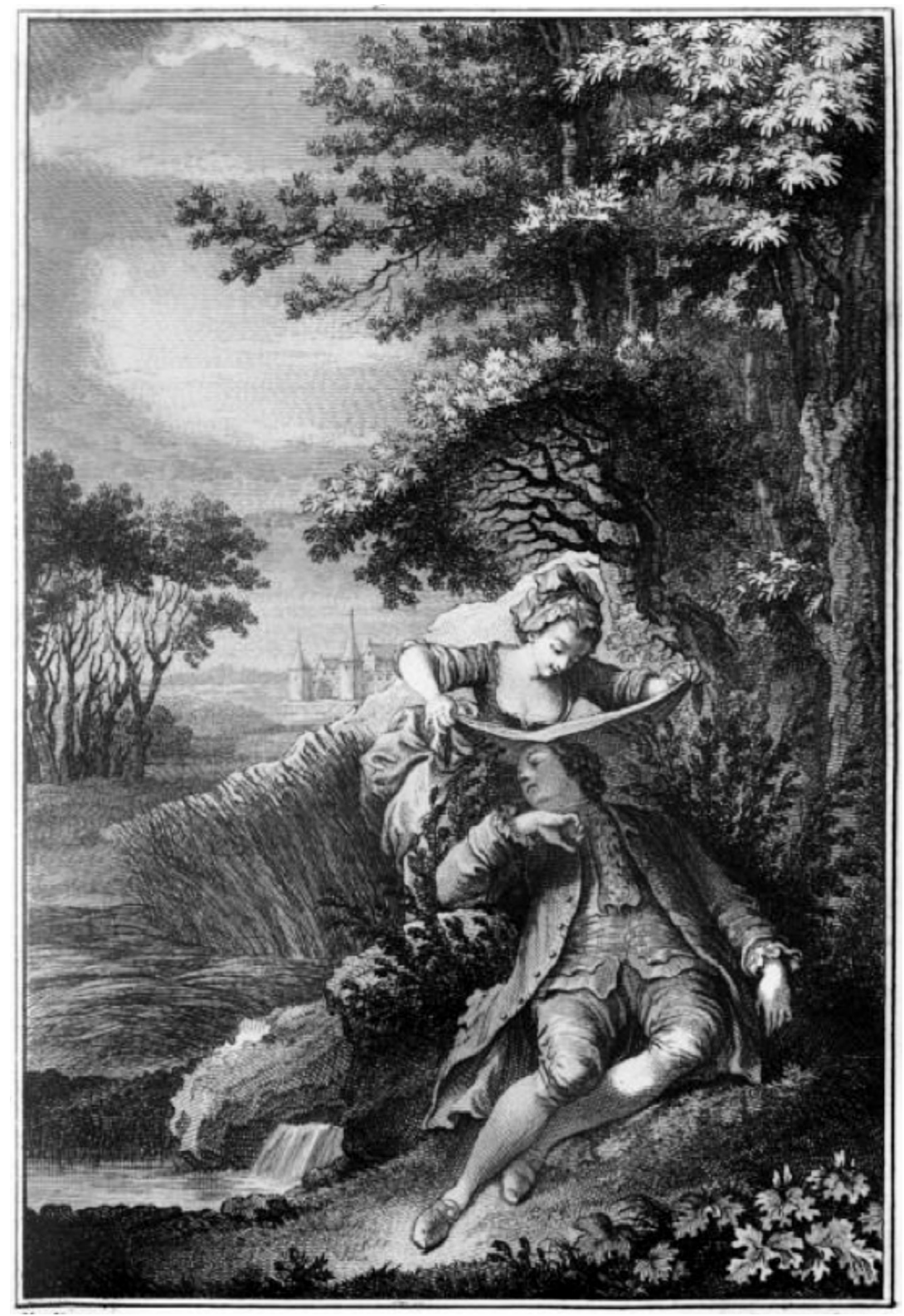

Fig. 6: Emmanuel Jean Nepomucène de Ghendt d'après Charles Eisen, Les moissonneurs, Acte III, gravure, dans Charles-Simon Favart, Théâtre ou Recueil des comédies, parodies E opéra-comiques..., Paris, Duchesne, 1772, t. X, en regard de la p. 53. 
sommeil et dans celui de son âge de façon que je suis arrivé jusqu’à son lit sans qu'elle se soit réveillée. J'ai d'abord été tenté d'aller plus avant et d'essayer de passer pour un songe; mais, craignant l'effet de la surprise et le bruit qu'elle entraîne, j’ai préféré d'éveiller avec précaution la jolie dormeuse $^{44}$.» Utilisant tour à tour conviction et menace, Valmont parviendra à ses fins.

Cependant, il serait hâtif de conclure que la voyeuse reste toujours prude. Ayant atteint l'âge de raison ou de «discrétion » - tel que le qualifie l'Église - elle est plutôt encline à se faire de plus en plus indiscrète. Ainsi La curieuse de Charles-Emmanuel Patas ${ }^{45}$ semble peu disposée à prendre en compte l'avertissement inscrit en bas de la gravure qui lui intime l'ordre de ne pas aller plus avant dans la découverte du «dangereux mystère » qui se cache sous le chapeau du berger endormi, judicieusement placé à l'entrejambe. Le sommeil n'est d'ailleurs pas impérativement requis pour ces expériences d'anatomie. Les jeunes paysannes de Jean-François Janinet ${ }^{46}$ sont suffisamment dégourdies pour entreprendre la même aventure sous l'œil complaisant de l'intéressé, tandis que l'élégante de Lavreince ${ }^{47}$ - tout aussi délurée que ses consœurs - préfère néanmoins se tourner vers un Priape figé dans son sommeil de pierre. On notera d'ailleurs que c'est seulement vers 1790 que le mot «déluré » se forme, sans aucun doute après une pratique constante de l'observation curieuse permettant de ne plus se laisser prendre au leurre.

Le voyeur masculin peut commencer son éducation très jeune, comme l'indique une gouache de Baudouin ${ }^{48}$ qui montre, selon Edmond de Goncourt: «Un gouverneur pénétrant avec son élève dans une chambre à coucher, où se voit, sur un lit, une femme dormant presque nue ${ }^{49}$.» Mais si, à la ville, les libertins commencent leur carrière plus rapidement que leurs consœurs fraichement sorties du couvent, souvent, les filles des campagnes s'éduquent plus vite

44. Pierre-Ambroise-François Choderlos de Laclos, op. cit., p. 201.

45. D'après Louis Chauveau, La curieuse, 1764-1789, gravure, marché de l'art.

46. D’après Jean-Jacques Lagrenée, L'oiseau privé, 1780-1790, gravure, 49,5 × $38,5 \mathrm{~cm}$, Londres, British Museum.

47. Jean-François Janinet d'après Nicolas Lavreince, Ah! Laisse-moi donc voir, 1780-1790, gravure, Washington, National Gallery of Art.

48. Le matin, avant 1769, dessin, 25,9 × 19,1 cm, New York, Metropolitan Museum.

49. Edmond de Goncourt, La maison d'un artiste, Paris, Charpentier, 1881, t. I, p. 44 . 


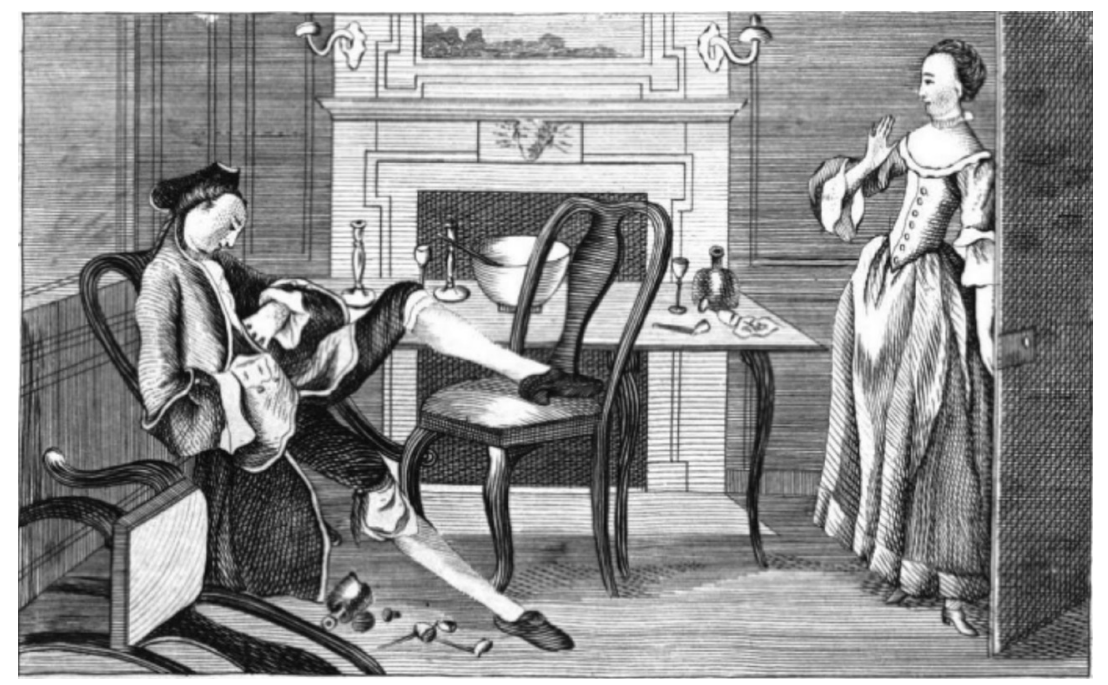

Fig. 7: Anonyme, gravure, dans John Cleland, Nouvelle traduction de Woman of Pleasur ou Fille de joye, Londres, Fenton, 1770, en regard de la p. 34.

que les rustauds. Fanny n'a que quatorze ans mais elle sait ce qu'elle contemple quand elle tombe sur Charles endormi, « un garçon de dixhuit à dix-neuf ans, fait au moule, \& beau comme les Anges ${ }^{50} »$ (Fig. 7), nous dit John Cleland dans La fille de joye. Les gravures anonymes qui accompagnent les nombreuses versions du texte peuvent devenir fort explicites à ce sujet. En revanche Saturnin, avant d'entamer une grande carrière de débauché, doit être déniaisé et y éprouve tout d'abord quelques difficultés décrites par Gervaise de Latouche et illustrées par Elluin ${ }^{51}$. Alors que Mme Dinville semble assoupie, Saturnin s'enhardit et essaye de "pénétrer dans l'obscur pays de l'amour » mais n’y voit rien car, dit-il: «ses jambes étaient croisées et la cuisse droite se trouvant collée sur la gauche, mettait mes regards en défaut ${ }^{52}{ }$. La mention de ces deux œuvres nous amène inévitablement aux portes

50. John Cleland, Nouvelle traduction de Woman of Pleasur ou Fille de joye, Londres, Fenton, 1770 [1749], t. I, p. 34.

51. François-Rolland Elluin, Saturnin et Mme Dinville, gravure $n^{\circ} 7 / 16$, dans Jean-Charles Gervaise de Latouche, Histoire de dom B****; portier des Chartreux, Rome [Londres], Philotanus, 1750 [1741].

52. Jean-Charles Gervaise de Latouche, op. cit., p. 75. 
de l'Enfer de la Bibliothèque nationale de France où il est bien rare de trouver des voyeurs et des voyeuses contemplant des personnages endormis ${ }^{53}$. L'illustration pornographique préfère montrer des couples en pleine action, scènes dans lesquelles il reste néanmoins à noter que la voyeuse est tout aussi présente que le voyeur, que l'on pense à Thérèse philosophe, Monrose, La fille de joie, Le rideau levé ou Les heures de Paphos pour les voyeuses et à L'histoire de Dom Bougre, Les écarts du tempérament, Hipparchia, Le doctorat impromptu, Mon noviciat ou Les joies de Lolotte, Le paysan perverti et Les lauriers ecclésiastiques pour les voyeurs. Dans la «fermentation discursive ${ }^{54} »$ du XviII ${ }^{\mathrm{e}}$ siècle, on sait à quel point les frontières entre les ouvrages estampillés comme libertins, érotiques, pornographiques ou obscènes sont poreuses, ceux-ci ayant été notamment analysés par Raymond Trousson ${ }^{55}$ et Jean-Marie Goulemot $^{56}$. Mais le propos n'est pas, ici, d'étudier l'illustration pornographique qui se distingue de la gravure érotique par le fait qu'elle représente le moment de l'accouplement, alors que l'œuvre érotique suspend l'action dans le moment qui précède ou illustre le moment qui suit. C'est le moment qui précède l'acte qu'illustre la gravure de Monnet dans laquelle une innocente, volontairement maintenue dans cet état par sa mère, surprend cette dernière soupirant d'aise dans les bras de son amant (Fig. 8): Voltaire conclue avec humour que la fille s'en trouvera déniaisée et prendra elle aussi un amant. Et c'est sur le «moment d'après » que s'attarde la gravure de Beauvarlet: surprenant un couple voluptueusement endormi, un magistrat semble tirer parti de l'occasion pour pontifier paisiblement sur les mérites comparés des élans du cour face à l'institution du mariage qui met Lamour à l'épreuve ${ }^{57}$. Précisons que la représentation de l'adultère intéresse très peu le XviII ${ }^{\mathrm{e}}$ siècle ${ }^{58}$ qui dévolue la contemplation du «moment

53. On notera qu'il n'y a pratiquement pas de voyeur chez Sade car le boudoir fonctionne comme un panoptique érotique, comme le signale Marcel Henaff (Sade. L'invention du corps libertin, Paris, Presses universitaires de France, 1978, p. 125).

54. Michel Foucault, Histoire de la sexualité: la volonté de savoir, Paris, Gallimard, 1976 , t. I, p. 26.

55. Raymond Trousson, Romans libertins du XVIII siècle, Paris, Laffont, 1993.

56. Jean-Marie Goulemot, Ces livres qu'on ne lit que d'une main. Lectures et lecteurs de livres pornographiques au XVIII ${ }^{e}$ siècle, Aix-en-Provence, Alinea, 1991.

57. Jacques-Firmin Beauvarlet d'après Pierre-Antoine Baudoin, Lamour à l'épreuve, 1777 , gravure, $37,2 \times 26 \mathrm{~cm}$, Paris, Musée du Louvre.

58. Voltaire estime qu'il s'agit d'«un crime que tout le monde est tenté de commettre, que toute le monde favorise quand il est commis; qu'il est difficile de prouver, 


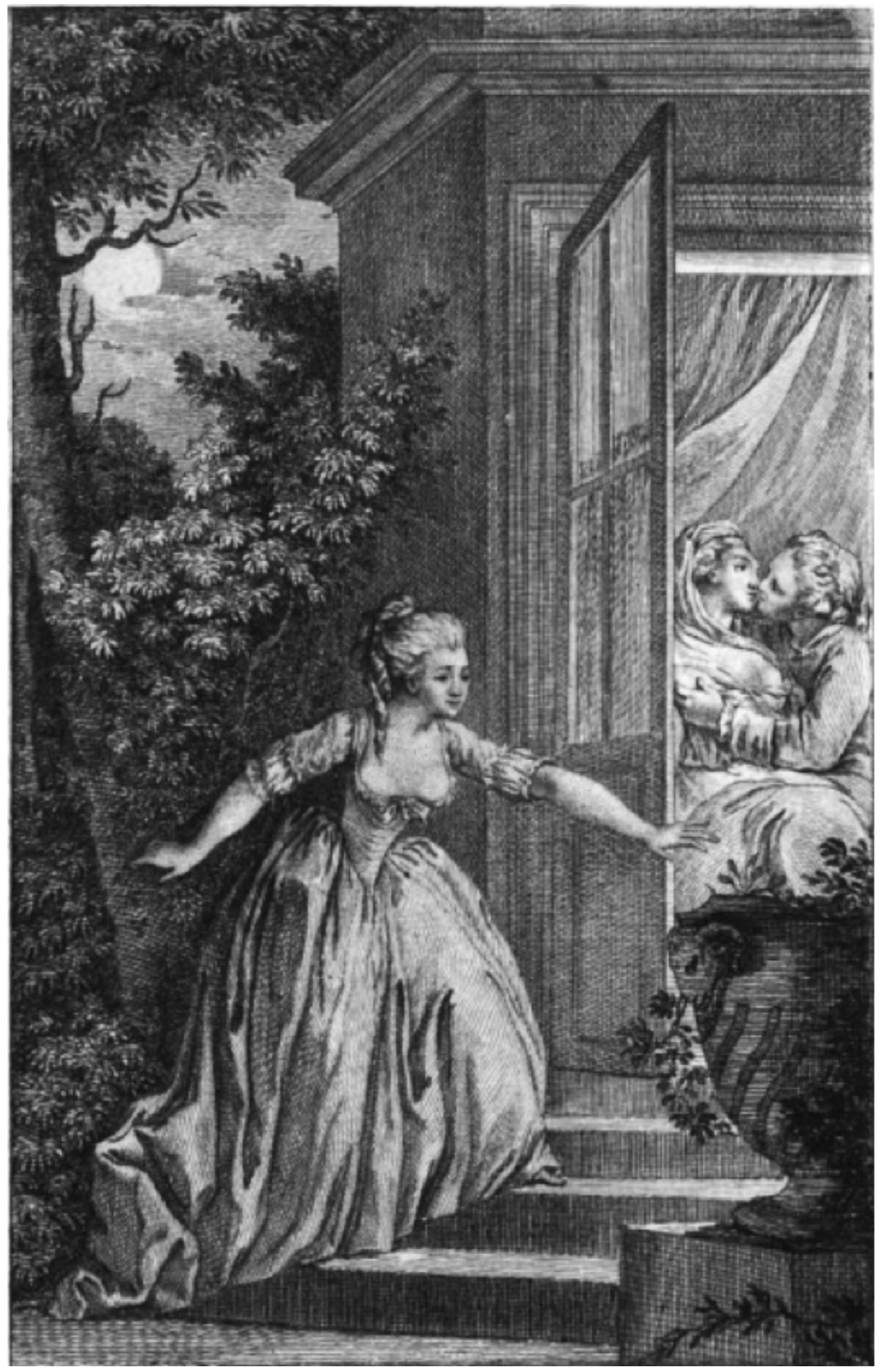

Fig. 8: Gérard Vidal d'après Charles Monnet, Gertrude ou L'éducation d'une fille, gravure, dans François-Marie Arouet dit Voltaire, Romans et contes, Bouillon, Société typographique, 1778, t. III, en regard de la p. 188. 
d'après » aux infortunés parents découvrant - trop tard - que leur surveillance a été trompée et qu'il faut marier au plus vite la fautive béatement endormie dans les bras de son amant, sujet qu'évoque la gravure de Jean-Louis Anselin d'après Antoine Borel intitulée judicieusement Vous avez la clef, mais il a trouvé la serrure ${ }^{59}$.

\section{La feinte du dormeur}

Si voyeurs et voyeuses s'enhardissent facilement auprès du dormeur ou de la dormeuse, c'est qu'il pèse toujours sur ces endormis une suspicion: ils feraient semblant de dormir. Dès 1694, le Dictionnaire de l'Académie française ${ }^{60}$ prend pour exemple de la feinte le «faire semblant de dormir ». Cet exemple sera repris tout au long du siècle dans les différentes éditions du Dictionnaire de l'Académie.

Le leitmotiv de la belle endormie qui feint de l'être pour attirer à elle un amant trop frileux est fréquemment mis en scène. Ainsi, dans La chercheuse d'esprit de Favart, Nicette fait-elle semblant de dormir pour qu'Alain puisse lui déclarer sa flamme, et tous deux trouveront ensemble l'esprit cherché (Fig. 9). Dans la gravure de Jean Dambrun d'après François-Marie Isidore Queverdo ${ }^{61}$, le voyeur soulève le drap qui recouvre la dormeuse, tandis que celle-ci se découvre complaisamment pour le bénéfice de celui qui va certainement interrompre son sommeil, mais aussi pour celui de cet autre voyeur de la scène qu'est le propriétaire de la gravure. Le sourire entendu de la dormeuse laisse penser qu'elle a le sommeil bien léger malgré ses yeux fermés.

Du côté de la voyeuse, le «faire semblant» du dormeur jouit d'une fortune identique. Celle-ci est soutenue et réanimée par les innombrables représentations de la fable du Mazet de Lamporecchio présentée soit dans la version de Boccace, soit dans celle de La Fontaine, et vendue soit comme partie intégrante du livre, soit au feuillet. La scène

et dont on ne peut guère se plaindre en justice sans se couvrir de ridicule. La société a fait une convention secrète de ne point poursuivre des délits dont elle s'est accoutumée à rire», dans "Prix de la Justice et de l'Humanité », Gazette de Berne, XIV, 15 février 1777 .

59. Washington, National Gallery of Art.

6o. Le dictionnaire de l'Académie française dédié au Roy, Paris, Veuve Coignard, 1694 , t. II, Art. «Semblant».

61. Le sommeil interrompu, gravure, 1787 , Washington, National Gallery of Art. 


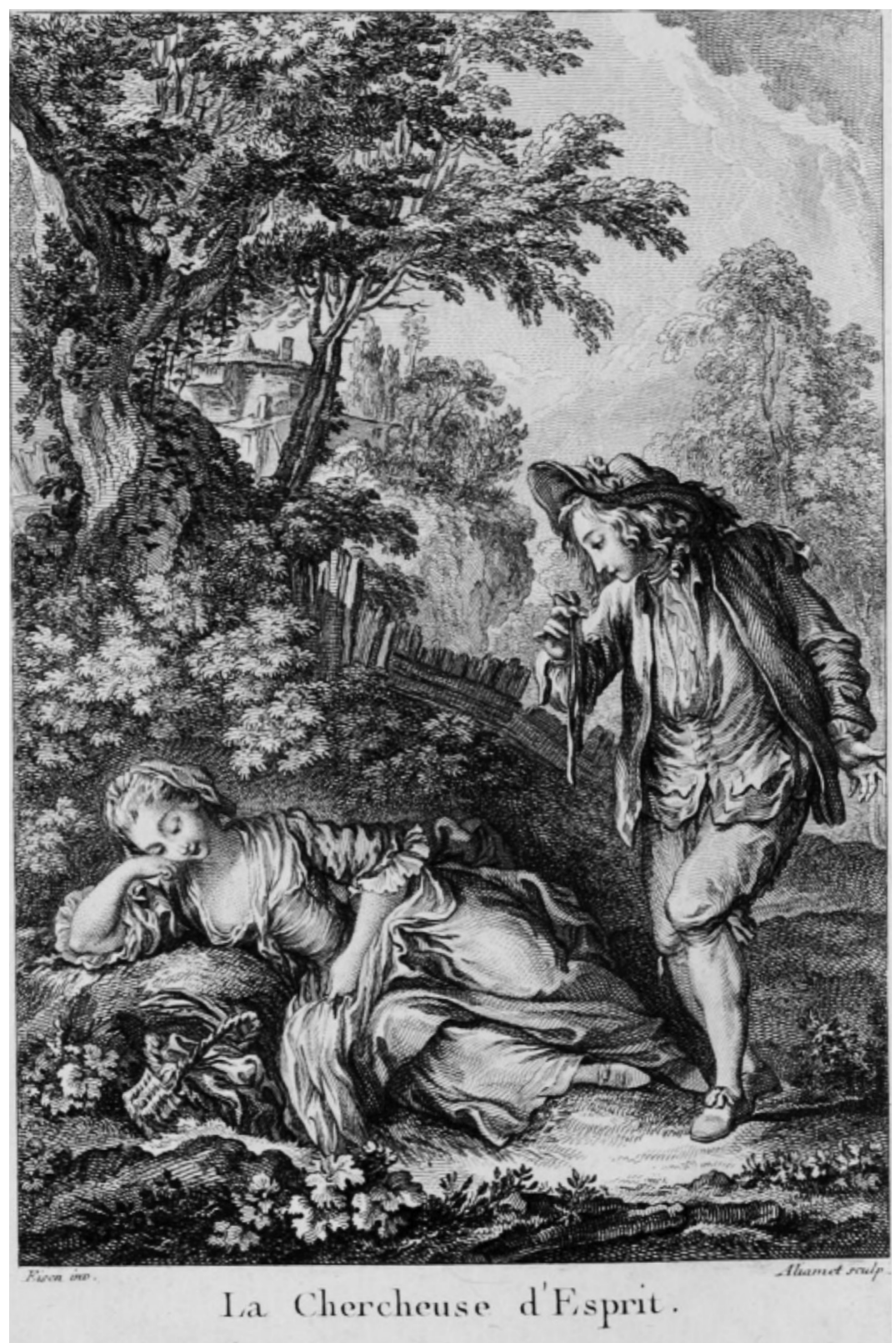

Fig. 9: Jacques Aliamet d'après Charles-Dominique-Joseph Eisen, La chercheuse d'esprit, gravure, dans Charles-Simon Favart, Théâtre ou Recueil des comédies, parodies É opéra-comiques..., Paris, Duchesne, 1743, t. VI, en regard de la p. 44. 
est représentée en 1757 et 1779 par Gravelot (Fig. 10), en 1762 par Eisen $^{62}$ pour l'édition des Contes de La Fontaine dite des Fermiers généraux, vers 1765-1777 par Fragonard ${ }^{63}$ pour un projet d'illustration des Contes, et encore en 1802 par Marillier ${ }^{64}$. Nul doute que cette fable ne soit à l'origine de petits tableaux de cabinet qui semblent y faire indirectement allusion aussi bien en France, avec Jean-Baptiste Besnard $^{65}$, qu'en Angleterre avec Philippe-Jacques de Loutherbourg ${ }^{66}$. À Londres, Francis Hayman peint un tableau - aujourd'hui disparu - sur un sujet similaire dans lequel on peut voir un essaim de jeunes femmes s'enhardir auprès d'un homme opportunément endormi sur une banquette, le sourire aux lèvres. Le tableau faisait partie d'une série de peintures destinées à orner les salons du Vauxhall londonien. Les nombreuses gravures ${ }^{67}$ reproduisant le tableau témoignent du succès de ce sujet quelque peu licencieux. En France, le même thème est repris et amplifié par Restif de la Bretonne dans ses Parisiennes ${ }^{68}$. Jovienne de Sanerville, croyant son amant mort à la suite d'un duel, devient folle de tristesse et remplace l'être aimé par une poupée. Mais l'amant gravement blessé se rétablit et vient subrepticement prendre la place de cette poupée. Faisant alors semblant de dormir - ce que montre la gravure de Louis Binet accompagnant le texte -, il revient progressivement à la vie et Jovienne, à la raison.

62. Charles-Étienne Gaucher d'après Charles Eisen, Mazet de Lamporecchio, gravure dans Jean de La fontaine, Contes et nouvelles en vers, Paris, Barbou, 1762, t. II, p. 67 .

63. Jean-Honoré Fragonard, Mazet de Lamporecchio, 1765-1777, dessin, 20,4 $\times$ $14 \mathrm{~cm}$, Paris, Musée du Petit Palais.

64. Rémi Delvaux d'après Clément-Pierre Marillier, Mazet de Lamporecchio, 1802, gravure, dans Boccace, Nouvelles, traduction libre par Mirabeau, Paris, Egron, 1802 , t. I, p. 254.

65. Le Mazet, Conte de La Fontaine, 1751-1789, huile sur toile, $31 \times 37 \mathrm{~cm}$, marché de l'art.

66. Le berger endormi et les deux bergères, 1760-1789, huile sur cuivre, 28,9 $\times$ $23,3 \mathrm{~cm}$, marché de l'art.

67. Louis Truchy d'après Francis Hayman, Stealing a Kiss ou Le baiser dérobé, 1743, gravure, $25,7 \times 36 \mathrm{~cm}$, Londres, British Museum.

68. Nicolas-Edme Restif de La Bretonne, Les Parisiennes. Les jeunes filles et les filles à marier, ou XL caractères généraux pris dans les mours actuelles, propres à servir à l'instruction des personnes-du-sexe. Tirés des mémoires du nouveau "Lycée des moeurs », Neufchâtel/Paris, Guillot, 1787, t. I, La folle, p. 275-295. 


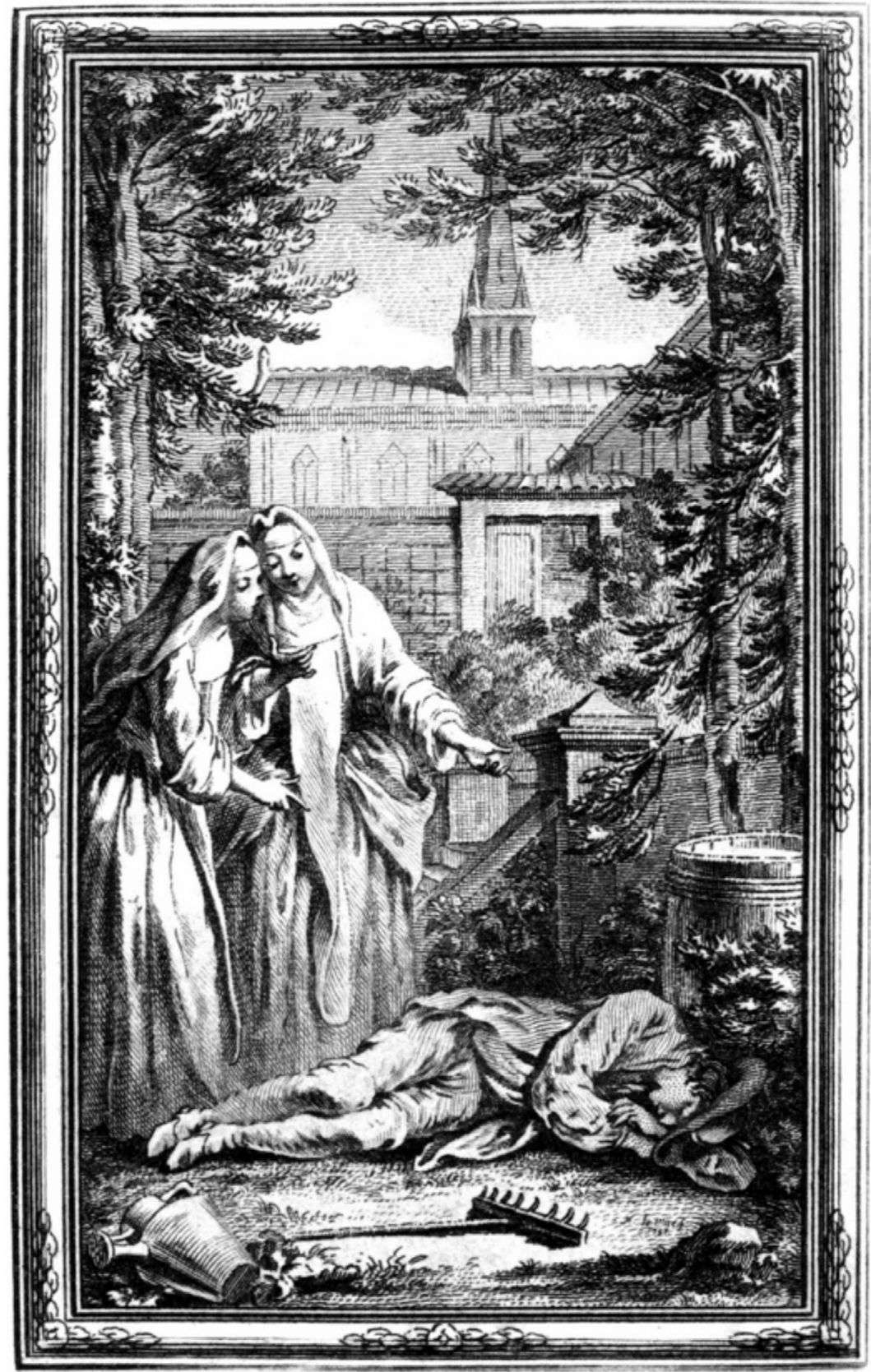

Fig. 10: Noël Lemire d'après Hubert-François Bourguignon dit Gravelot, Mazet de Lamporecchio endormi au jardin, gravure, dans Boccace, Il Decamerone di M. Giovanni Boccacio, Londra [Paris], s. n., 1757, t. II. nº 3 . 
À la fin du XVIII ${ }^{e}$ siècle, Boilly réinterprète le sujet du «faire semblant» sous la forme narrative de deux tableaux en pendant qui décrivent l'intimité du couple. Dans Le sommeil trompeur ${ }^{69}$, un jeune homme en négligé du matin feint de dormir, le bras droit appuyé sur un clavecin ouvert. La jeune femme tente de lui enlever le livre qu'il tient encore de la main gauche, mais, dans le pendant ${ }^{70}$, le dormeur s'est réveillé et enlace la jeune femme par la taille, refermant ainsi sur elle le piège amoureux dans lequel elle est tombée.

Ce parcours - forcément voyeuriste - parmi les voyeurs et les voyeuses du XVIII ${ }^{\mathrm{e}}$ siècle, montre que cette activité n'est pas réservée à la seule gente masculine, mais peut aussi être pratiquée par des femmes curieuses. Les sujets de leurs observations ne sont pas aussi étendus que ceux de leurs confrères voyeurs mais restent, néanmoins, proches. De même, leurs réactions face au spectacle proposé, sont similaires à celles des hommes, tout en échappant à l'éventuelle violence masculine qui peut suivre le voyeurisme. Comme les voyeurs, les voyeuses n’affichent ni pruderie excessive, ni attitude scandalisée, mais montrent leur vif intérêt face au spectacle proposé qui, participant de leur éducation, fait du voyeurisme un comportement assumé, un jeu joyeux et jouissif. Aussi pourrait-on nuancer les propos de Jean-Marie Goulemot qui affirme que «l'idéal féminin de ce siècle dit amoureux, c'est bien plus la Justine de Sade soumise aux assauts des libertins, que la conquérante Madame de Merteuil ${ }^{71}$ ». Entre ces deux figures de la féminité du second XvIII ${ }^{e}$ siècle, l'on pourrait intercaler la figure de la voyeuse, qui n'est ni victime, ni dominatrice, pas plus qu'elle n'est complaisante ou obsédée. Une voyeuse qui, à l'égal du voyeur, peut avoir les mêmes curiosités et les mêmes désirs.

Bien sûr, il ne faut pas négliger le fait que ces tableaux et gravures montrant des voyeuses prises de libido sciendi sont, majoritairement,

69. Louis-Léopold Boilly, Le sommeil trompeur, 1796, huile sur toile, $40 \times 32,5 \mathrm{~cm}$, marché de l'art.

70. Louis-Léopold Boilly, Le réveil prémédité, 1796, huile sur toile, $40 \times 32,5 \mathrm{~cm}$, marché de l'art.

71. Jean-Marie Goulemot, «Préface» de De la nymphomanie ou fureur utérine du Dr. Bienville [1771]. Suivi de quelques articles de l'Encyclopédie, Paris, Le Sycomore, 1980 , p. 10-11. 
faits par et pour un public masculin ${ }^{72}$. Mais, comme le montre le tableau de Louis-Léopold Boilly, intitulé Un jeune peintre et son modèle $e^{73}$, il peut y avoir une véritable connivence autour de la pratique assumée du voyeurisme. Le peintre et son modèle, assis sur les genoux de ce dernier, regardent ensemble l'œuvre que vient d'achever l'artiste: il s'agit d'une version sécularisée du mythe de Jupiter et Antiope dans laquelle un voyeur satyre a posé sa main sur le sein d'une femme endormie au premier plan. Au lieu d'être située en pleine nature, la scène se déroule dans l'intimité d'un boudoir ou d'une chambre. Le tableau fait retour sur les protagonistes de la scène qui se présentent comme les spectateurs de l'œuvre, mais qui en furent préalablement les acteurs. En tant que spectateurs, ils imagent une résolution souriante de la rencontre du satyre et de la nymphe et semblent indiquer que la nature sensuelle de leurs relations n'a plus besoin - en ce XVIII ${ }^{\mathrm{e}}$ siècle finissant - de se cacher derrière le voile de la Fable. En tant qu'acteurs du tableau, le peintre et son modèle ont endossé les rôles de satyre et de nymphe modernes, l'un observant longuement l'autre dormant ou faisant semblant de dormir. Ils illustrent ainsi non seulement la philosophie de Condillac ${ }^{74}$ qui accorde aux deux sens que sont la vue et le toucher une importance prééminente dans la connaissance, mais aussi et surtout la philosophie de La Mettrie. Pour ce dernier, la contemplation de l'amant ou de l'amante endormie permet de stimuler l'imagination et fait partie d'un processus par gradation ${ }^{75}$ permettant d'accéder au plus haut des plaisirs.

72. Notons néanmoins que, comme l'indique Patrick Michel (Peinture et plaisir. Les goûts picturaux des collectionneurs parisiens au XVIII siècle, Rennes, Presses Universitaires de Rennes, 2010, p. 102-104), il existe aussi des collectionneuses, même si elles restent pour la plupart largement méconnues, telles Madame de Gontaut ou les Demoiselles de Boissière, pour ne pas citer la comtesse de Verrue, la marquise de Pompadour, la comtesse du Barry ou encore Madame de Bandeville.

73. 1788-1792, huile sur toile, $46 \times 37,8 \mathrm{~cm}$, Northampton, Smith College Museum of Art. Pour Suellen Diaconoff (Through the Reading Glass: Women, Books, and Sex in the French Enlightenment, Albany, State University of New York Press, 2005), il s'agit d'une mise en abyme du désir qui serait aussi un texte dans un texte dupliquant l'acte de lecture de la femme désirante autant que désirée.

74. Etienne Bonnot de Condillac, Traité des sensations, Londres/Paris, De Bure, 1754,2 vol.

75. Peter Maxwell Cryle, La crise du plaisir 1740-1830, Lille, Presses universitaires du Septentrion, 2003; Michel Delon, Le savoir-vivre libertin, Paris, Hachette, 2004, p. 91-92. 
Ainsi, voyeurs et voyeuses participent-ils ensemble, et à parts égales, d'un certain Art de jouir ${ }^{76}$ propre au XVIII siècle, aujourd'hui disparu, comme le constate Foucault ${ }^{77}$, au profit d'une simple scientia sexualis.

76. Julien Offray de La Mettrie, L'art de jouir, Cythère, s. n., 1751, p. 30 : «Belles, qui voyez vos Amants s'endormir sur votre beau sein, si vous êtes curieuses d'essayer le transport d'un Amant assoupi, restez éveillées, s'il vous est possible; le même cœur, soyez-en sûres, la même âme vous communiquera les mêmes feux: feux d'autant plus ardents, qu'il ne sera pas distrait de vous par vous-même. Il soupirera dans le fort de sa tendresse, il parlera même \& vous pourrez lui répondre; mais que ce soit très doucement...»

77. Michel Foucault, op. cit., p. 76-84, chap. III Scientia sexualis. Foucault oppose l'ars erotica (une culture des rites d'initiation aux plaisirs pluriels) à la scientia sexualis (une nature physiologique et une mécanique de crise du plaisir vécue au singulier). 